\title{
Short communication: Dietary bovine milk-derived exosomes improve bone health in an osteoporosis-induced mouse model
}

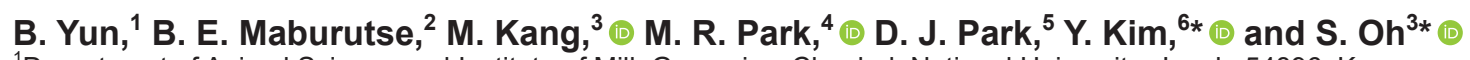 \\ ${ }^{1}$ Department of Animal Science and Institute of Milk Genomics, Chonbuk National University, Jeonju 54896, Korea \\ ${ }^{2}$ Department of Animal Sciences and Health, Marondera University of Agricultural Sciences and Health, PO Box 35, Marondera, Zimbabwe \\ ${ }^{3}$ Department of Functional Food and Biotechnology, Jeonju University, Jeonju 55069, Korea \\ ${ }^{4}$ Department of Pharmacology and System Physiology, University of Cincinnati, OH 45267 \\ ${ }^{5}$ Korea Food Research Institute, Jeollabuk-do 55365, Korea \\ ${ }^{6}$ Department of Agricultural Biotechnology and Center for Food and Bioconvergence, and Research Institute of Agriculture and Life Science, \\ Seoul National University, Seoul 08826, Korea
}

\begin{abstract}
Osteoporosis is a systemic skeletal disease characterized by low bone mass and micro-architectural deterioration of bone tissue, with a consequent increase in bone fragility and fracture susceptibility. In an aged society with increased life expectancy, the incidence rate of osteoporosis is also rapidly increasing. Inadequate nutrition may negatively influence bone metabolism. Recently, many studies have investigated the functionality of milk-derived exosomes, which play important roles in cell-to-cell communication. However, there are few reports of how milk-derived exosomes influence osteoblast proliferation and differentiation. Here, we determined whether bovine colostrum-derived exosomes promote anti-osteoporosis in vitro and in vivo. Tartrate-resistant acid phosphatase-stained cells were significantly inhibited in Raw264.7 cells treated with exosomes, indicating reduced osteoclast differentiation. We induced osteoporosis in mice using glucocorticoid pellets after orally administering exosomes for 2 mo. Interestingly, the bone mineral density of exosome-fed mouse groups was significantly improved compared with the glucocorticoid-induced osteoporosis group without exosome treatment. In addition, Lactobacillus were decreased in the gut microbiota community of osteoporosis-induced mice, but the gut microbiota community composition was effectively restored by exosome intake. Taken together, we propose that exosomes isolated from bovine colostrum could be a potential candidate for osteoporosis prevention, bone remodeling improvement, and inhibition of bone resorption. To our knowledge, this is the first time that a protective effect
\end{abstract}

Received August 27, 2019.

Accepted April 14, 2020.

$\mathrm{kr}$

*Corresponding authors: osangnam@jj.ac.kr and ykeys2584@snu.ac of milk exosomes against osteoporosis has been demonstrated in vivo. Our results strongly suggest that bovine colostrum exosomes might be used as a prophylaxis to prevent the onset of osteoporosis. Indeed, our results offer promising alternative strategies in the nutritional management of age-related bone complications.

Key words: milk-derived exosome, osteoporosis, osteoclast differentiation, microbiota

\section{Short Communication}

Osteoporosis is a skeletal disorder resulting in reduced bone strength, with high prevalence in women aged 50 to 69 yr (NIH Consensus Development Panel on Osteoporosis Prevention, Diagnosis, and Therapy, 2001). In particular, osteoporotic fractures are most common in postmenopausal women or elderly people, and are a major cause of global health spending (Leder et al., 2015). Although many biological, nutritional, and behavioral factors contribute to osteoporosis, age, bone mass loss, structural deterioration, and frequency of falls eventually increase (Weaver et al., 2016). Milk and dairy product supplementation is widely recommended to prevent osteoporosis and subsequent fractures because various functional ingredients, including milk basic protein, casein phosphopeptide, and lactoferrin, are beneficial for bone health (Uenishi, 2006). In particular, colostrum has been studied extensively as milk containing nutrient-rich, immune, developmental, and tissue-repairing factors produced in the mammary glands shortly after giving birth (Uruakpa et al., 2002). Several studies show that colostrum intake is beneficial to bone health and colostrum is an important functional substance for bone health (Batty and Bionaz, 2019).

Exosomes are membranous vesicles $(30-150 \mathrm{~nm})$ of endocytic origin. These extracellular vesicles $(\mathbf{E V})$ are natural nanoparticles that play an important role in cell-to-cell communication. Extracellular vesicles medi- 
ate intercellular communication via delivery of various proteins, lipids, and RNA (Zempleni et al., 2019). Exosomes are present in all biofluids (blood, milk, saliva, urine, and so on) and are considered to be new players in cell-to-cell communication (Bang and Thum, 2012). Some studies report that proteins present in milkderived exosomes have biological roles that include immune regulation, cell growth, and repair (Samuel et al., 2017). Interestingly, exosomal microRNA might be transferred from the mother to the infant by breastfeeding, providing an immune-related function (Zhou et al., 2012). Further, milk-derived exosomes have beneficial effects and numerous functions for aged people and infants (Bang and Thum, 2012; Zhou et al., 2012; Arntz et al., 2015; Samuel et al., 2017; Zempleni et al., 2019). Fortunately, bovine milk contains high exosome levels in both mature milk and colostrum, which suggests that exosomes containing microRNA and proteins could be good nutritional sources to research to develop functional food supplements. Arntz et al. (2015) showed that bovine milk-derived exosomes orally administered improved experimental arthritic symptoms in mice.

Studies of intestinal microbial changes according in various food and nutritional conditions provide a basic knowledge for future investigations of how interactions between food components and gut microbiota may influence or even determine human health and disease (Ercolini and Fogliano, 2018; Danneskiold-Samsøe et al., 2019). In addition, Ibáñez et al. (2019) reported that intestinal microorganisms affect bone health, and restoring the balance of intestinal microorganisms can be a treatment for various diseases. Based on these studies, we hypothesized that milk-derived exosomes isolated from colostrum could prevent osteoporosis. In this study, we investigated whether the exosomes isolated from bovine colostrum could delay the progress of osteoporosis, and how exosomes change the gut microbiota in osteoporosis-induced mice.

Bovine colostrum-derived exosomes (BCE) were isolated from bovine colostrum, which was individually collected from 10 dairy cows within $24 \mathrm{~h}$ of calving. Colostrum samples were pooled, and aliquots were prepared using a method previously described (Maburutse et al., 2017). Briefly, we sequentially centrifuged colostrum to defat the colostrum, pellet the cell debris, and make a whey fraction, which was then ultracentrifuged at $100,000 \times g$ for $60 \mathrm{~min}$ at $4^{\circ} \mathrm{C}$. We collected the supernatant and ultracentrifuged it at $135,000 \times g$ for 90 min at $4^{\circ} \mathrm{C}$ to pellet the exosomes. The pellets were then resuspended in PBS or saline, filtered through $0.22-\mu \mathrm{m}$ filters, and frozen for later use. To verify the authenticity of the exosome samples we separated, we conducted microscopy, particle size analysis, and Western blotting for exosomal-specific markers (Figure 1). The exosome particle shape was determined by scanning electron microscopy and transmission electron microscopy. The extracted exosomes were round and had double membranes, confirming their identity (Figure 1A). For scanning electron microscopy imaging, exosome particles were fixed in $2 \%$ paraformaldehyde for 10 min at room temperature. Drops of fixed samples were placed on thoroughly dried silicon chips and air-dried before imaging. The exosome particle shape was confirmed using field-emission scanning electron microscopy (FE-SEM, Hitachi S-5500, Hitachi High Technologies, Tokyo, Japan). We also observed the shape and size of the exosome structure by transmission electron microscopy (Hitachi H-7650, Hitachi High Technologies). The particle size in exosome samples formed a single distribution with an average diameter of $75.7 \mathrm{~nm}$ (Figure 1B). Western blot analysis confirmed that exosome samples contained proteins commonly associated with exosomes (Kumar et al., 2015). For immunoblotting, we used mouse anti-CD9 (sc-13118), CD63 (sc-365604), and Tsg101 (sc-7964) antibodies (Santa Cruz Biotechnology, Santa Cruz, CA). Samples were lysed with ice-cold radioimmunoprecipitation assay buffer (Thermo Fisher Scientific, Waltham, MA) supplemented with protease and phosphatase inhibitors. The protein concentration was determined using a Bradford Protein Assay kit (Bio-Rad, Hercules, CA), and $30 \mu \mathrm{g}$ of whey. The BCE samples were resolved by SDS-PAGE. Proteins were transferred to Bio Trace PVDF membranes (Pall Life Sciences, Pensacola, FL) and detected using Pierce ECL Western Blotting Substrate (Life Technologies, Carlsbad, CA). Tetraspanins were highly detected in $\mathrm{BCE}$ compared with the whey fraction, whereas no difference was observed in the overall protein amount (visualized by Coomassie blue staining, Figure 1C).

In bone disease, decreased bone density is caused by an imbalance between bone formation and bone resorption driven by excessive osteoclast activity (Soysa and Alles, 2016). Therefore, it is important to control excessive osteoclast activity to maintain normal bone function. Receptor activator of nuclear factor kappaB ligand (RANKL) plays a crucial role in inducing osteoclastogenesis from hematopoietic cells of the monocyte-macrophage lineage. The RANKL-induced differentiation into osteoclasts is characterized by the formation of multinuclear giant cells, which generate the F-actin loop structure on the bone surface before bone resorption (Hayman, 2008).

We treated MC3T3-E1 preosteoblast cells with $20,50,100,150,300$, or $500 \mathrm{ng} / \mathrm{mL} \mathrm{BCE} \mathrm{to} \mathrm{evalu-}$ ate cytotoxicity and proliferation. Preosteoblasts were exposed to intact and ultrasonicated (to disrupt exosome structure) BCE. We did not detect cytotoxicity at any exosome concentration. Intact BCE facilitated cell 
(A)
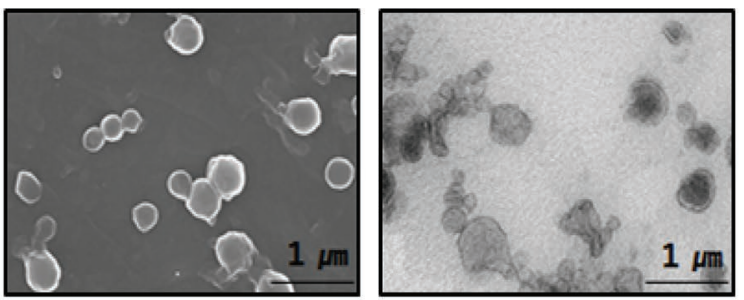

(B)

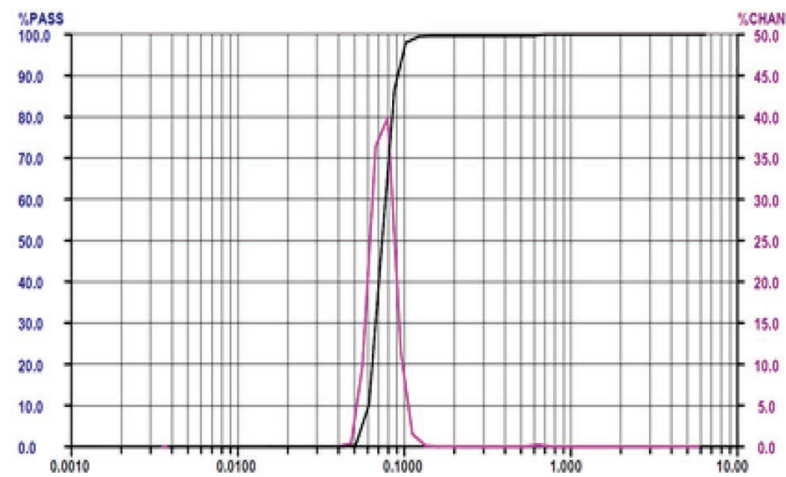

(C)

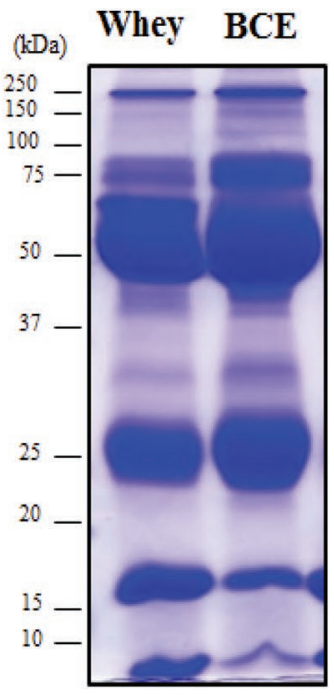

Whey BCE

\section{CD63 (34-55kDa)}

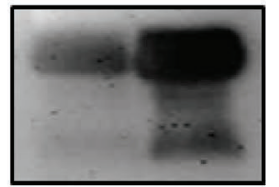

Tsg101

(48 kDa)

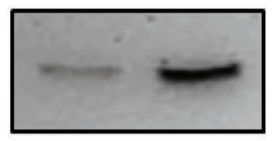

CD9 (26 kDa)

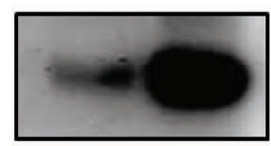

Figure 1. Bovine milk-derived exosome protein markers. (A) Scanning electron microscope (left) and transmission electron microscope (right) images of exosomes isolated from colostrum. (B) The particle size distribution in exosome samples was determined by dynamic light scattering (particle size analyzer, NanoPlus, Boulder, CO). PASS = accumulating passing percentage, black line; CHAN = percentage of particles at size (channel). (C) Protein profiles were analyzed by 12\% SDS-PAGE electrophoresis and Coomassie blue staining (left). Equal protein amounts $(30 \mu \mathrm{g})$ were loaded in the lanes of each gel. Immunoblotting for exosomal markers (CD9, CD63, and Tsg101) in whey were obtained after centrifuging at $100,000 \times g$, for $60 \mathrm{~min}$ at $4^{\circ} \mathrm{C}$, and precipitates (bovine colostrum-derived exosomes; BCE) were obtained after 135 ,000 $\times$ $g$ for 90 min at $4^{\circ} \mathrm{C}$ ultracentrifugation from colostrum, as described in Maburutse et al. (2017).

proliferation from $100 \mathrm{ng} / \mathrm{mL}$ (Supplemental Figure S1; https://doi.org/10.3168/jds.2019-17501). Sun et al. (2013) suggested that vesicle structure plays a biological role in cell entry. However, osteoblast differentiation was not verified.

To test whether BCE inhibit osteoclastogenesis, we stimulated RAW 264.7 cells with $50 \mathrm{ng} / \mathrm{mL}$ RANKL (R\&D Systems, Minneapolis, MN) and $25 \mathrm{ng} / \mathrm{mL}$ macrophage-colony-stimulating factor (R\&D Systems), according to previous studies (Zheng et al., 2006; Newa et al., 2011). We then exposed stimulated cells to high $(150 \mathrm{ng} / \mathrm{mL})$ or low $(50 \mathrm{ng} / \mathrm{mL})$ BCE concentrations and examined the cells with a Diagnostic Acid Phosphatase kit (Sigma, Castle Hill, Australia). The TRAP assay is the most common method to detect osteoclast induction and the degree of bone resorption in vitro (Ghayor et al., 2011). The TRAP-positive multinucleated cells $(\geq 3$ nuclei), which were considered to be osteoclasts, were imaged and counted using an optical microscope. The RANKL and macrophage-colonystimulating factor stimulated RAW 264.7 cell differentiation into TRAP-positive multinucleated osteoclasts in vehicle-treated controls, whereas the number of osteoclasts was significantly decreased in the presence of BCE (Figure 2A). In particular, high-level BCE (150 $\mathrm{ng} / \mathrm{mL}$ ) led to strong inhibition of osteoclast forma- tion. These results suggest that BCE inhibit osteoclast differentiation. These data suggest that BCE may play a role in decreasing bone resorption by disrupting osteoclast function.

Milk plays an important role in the growth of newborn animals. Indeed, bioactive components from milk can directly affect bone metabolism (Rani et al., 2017). Some studies suggest that a group of bioactive proteins derived from bovine whey prevent bone loss (Rani et al., 2017). Exosomes present in whey milk have many microRNA, which seem to have diverse beneficial activities for the host (Rani et al., 2017). After evaluating the effects of $\mathrm{BCE}$ on osteoclast differentiation in vitro, we investigated whether BCE is beneficial for osteoporosis in an animal model. All animal work was reviewed and approved by the Institutional Animal Care and Use Committee of Jeonju University (JJUIACUC-2018-01). To evaluate the effect of BCE consumption on the progression of osteoporosis, BCE were resuspended in saline and orally administered for 8 wk before osteoporosis induction. Eight-week-old mice are estimated to be equivalent to approximately 4- to 5 -yr-old humans. Because it takes at least 4 wk for osteoporosis induction in mice by steroid treatment, we chose a longer duration ( $8 \mathrm{wk}$ ) of oral administration to prevent degenerative diseases such as osteoporosis. 
(A)

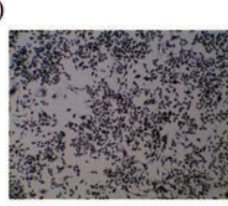

CTL

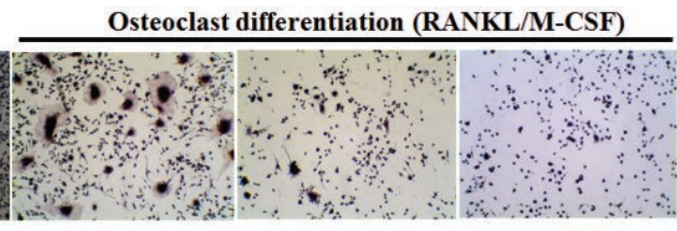

+ PBS

+ L-BCE

$+\mathrm{H}-\mathrm{BCE}$

(B)

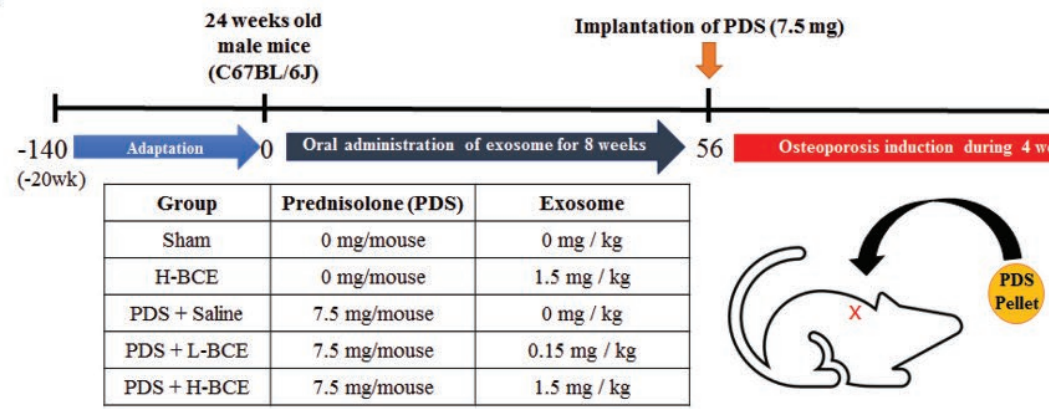

(C)

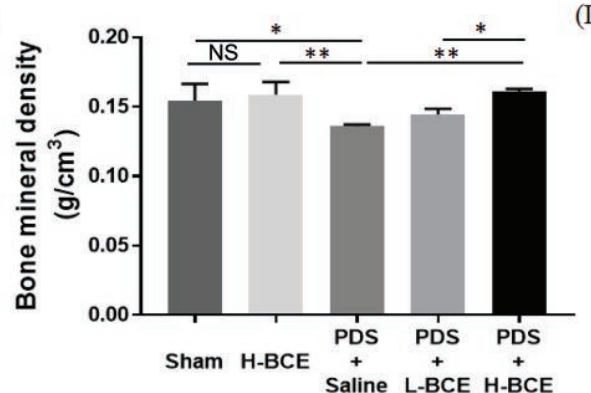

(D)

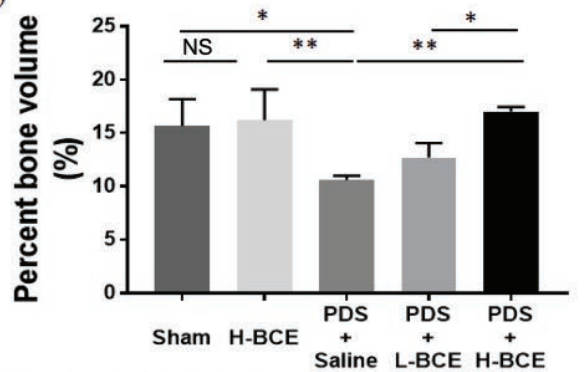

(E)

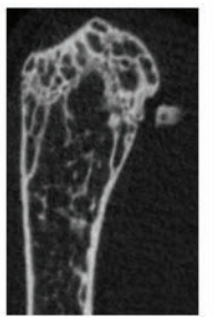

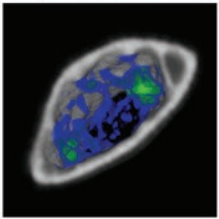

Sham
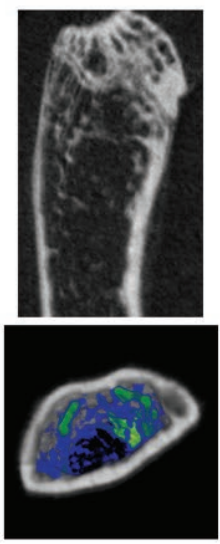

H-BCE
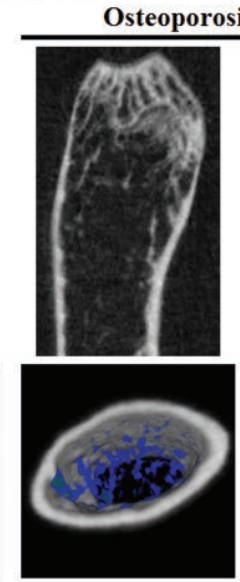

+Saline
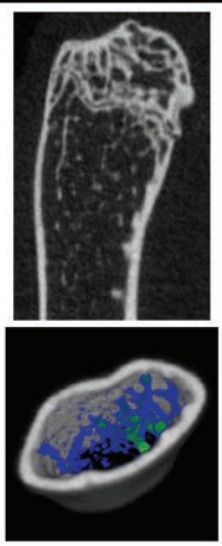

+L-BCE

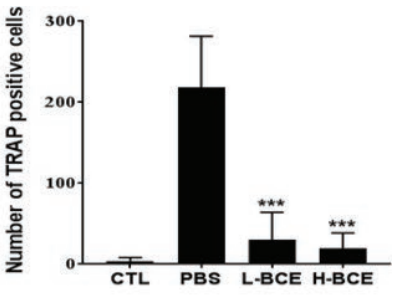

killed 
As in our preliminary study, our sample concentration at maximum was approximately $1.7 \mathrm{mg} / \mathrm{kg}$ for in vivo administration, which could be continuously isolated and did not cause significant changes in BW and intake (water, feed; Supplemental Figure S2; https://doi.org/ $10.3168 /$ jds.2019-17501); we determined $1.5 \mathrm{mg} / \mathrm{kg}$ as high dose and $0.15 \mathrm{mg} / \mathrm{kg}$ as low dose.

We induced osteoporosis using glucocorticoid treatment by placing slow-release pellets (PDS; $7.5 \mathrm{mg}$ prednisolone, Innovative Research of America, Sarasota, FL) subcutaneously on the shoulder area of the mice, according to the method published by Thiele et al. (2014). In these experiments, 60 mice (C57BL/6J, 4-wk-old males, BW: $16.9 \pm 2.2 \mathrm{~g})$ were divided into 5 groups [sham, high dose $(\mathbf{H})-\mathrm{BCE}, \mathrm{PDS}+$ saline, PDS + low dose (L)-BCE, and PDS + H-BCE]. Due to a difficulty of physical space, we divided 12 young mice into 2 cages (6 mice/cage, therefore, 2 cages/group) and raised at $24 \mathrm{wk}$ to prevent fighting. Sham mice (sham; $\mathrm{n}=12$ ) and osteoporotic mice (PDS; $\mathrm{n}=12$ ) were fed a normal diet without exosome intake. Before osteoporosis induction, experimental mice received $\mathrm{BCE}$ at a dose of $1,500 \mu \mathrm{g} / \mathrm{kg}$ (PDS + H-BCE; n = $12)$ or $150 \mu \mathrm{g} / \mathrm{kg}(\mathrm{PDS}+\mathrm{L}-\mathrm{BCE} ; \mathrm{n}=12)$ by oral gavage for 8 wk. Sham mice fed H-BCE $(n=12)$ were included investigate the biological effects of $\mathrm{BCE}$ on bone health and gut microbiota. These mice received only surgical treatment without implanted pellets. We scanned all the mice under anesthesia 4 wk after pellet implantation (Figure 2B). Raw tomographic data were acquired using a SkyScan-1076 micro-CT scanner (SkyScan, Aartselaar, Belgium) with the following conditions: pixel size, $35 \mu \mathrm{m}$; source voltage, $50 \mathrm{kVp}$; and source current, $200 \mu \mathrm{A}$. Micro-computed tomography (CT) imaging analysis was performed using software including NRecon reconstruction, CTAn 1.8, and CTvol (SkyScan, Aartselaar, Belgium). We converted volume-of-interest attenuation data to Hounsfield units and expressed these data as a bone mineral density (BMD) value using phantoms (SkyScan). Induction of osteoporosis was confirmed by visualizing the BMD of glucocorticoid implant mice compared with the sham group. Osteoporosis is associated with tissue loss in the cross-sectional area of the bone damage to the bone microstructure. In particular, the microstructure and biomechanical properties of the femur provide convincing evidence to explain the effect of compounds on osteoporosis (Frost et al., 1998). Femoral BMD and percent of bone volume were significantly $(P<0.05)$ lower in the PDS group than in the sham group (Figure $1 \mathrm{D}$ and $2 \mathrm{C}$ ). Osteoporotic mice fed a high BCE (PDS + $\mathrm{H}-\mathrm{BCE}$ ) showed higher BMD and percent of bone volume than osteoporotic mice not fed BCE (PDS). These results indicate that $\mathrm{BCE}$ ingestion significantly pre- vented glucocorticoid-induced osteoporosis $(P<0.01)$. Micro-CT images of trabecular bone of femurs for each BCE-fed group (Figure 2E), even osteoporotic groups, showed significantly high bone density. No significant difference was observed between the sham group and the H-BCE-fed group in this study. Diseases related to bone formation are often associated with milk consumption as a mitigating role in bone disease (Uenishi et al., 2007; Michaëlsson et al., 2014). Interestingly, EV from bovine milk induce higher osteocyte proliferation and increased osteoblast differentiation from mesenchymal stem cells (Oliveira et al., 2017). Our results suggest that $\mathrm{BCE}$ provide beneficial nutritional support for onset of osteoporosis.

The gut microbiome plays an important role in human health (Marchesi et al., 2016). Many studies reported that the gut microbiota is implicated in bone metabolism and disease (Sjögren et al., 2012; Guss et al., 2017). Several studies also reported that ingestion of exosomes isolated from milk changes the gut microbiota in a beneficial way (Zempleni et al., 2019). To test the hypothesis that the anti-osteoporosis activity of $\mathrm{BCE}$ is related to rebalancing the gut microbiota, we collected fecal samples from experimental mice. Samples were randomly pooled into 4 samples per group and stored at $-80^{\circ} \mathrm{C}$ until use. Metagenomic DNA was extracted from fecal samples (0.02 $\mathrm{g}$ per sample) using a QIAamp DNA Stool Mini Kit (Qiagen, Valencia, CA). We analyzed the16S rRNA V4 region (forward primer, 5'-CCTACGGGNGGCWGCAG-3'; reverse primer, 5'-GACTACHVGGGTATCTAATCC-3') using an Illumina MiSeq platform (Illumina, San Diego, CA). We used Mothur software (version 1.41) to analyze the raw sequencing data (Schloss et al., 2009). All sequence data were processed according to the Mothur standard operating procedure manual. We first removed sequencing errors and chimeras as previously reported (Pruesse et al., 2007; Rognes et al., 2016). Taxonomic classification was analyzed using Greengenes-formatted databases (2013 release; DeSantis et al., 2006), and eliminated sequences not categorized as archaea and bacteria. Singleton reads were removed using the split.abund Mothur subroutine (Unno, 2015). We classified operational taxonomic units with a 0.03 distance calculation (97\% sequence similarity). Then, we analyzed fecal microflora community diversity, using UniFrac analysis to compare the degree of microbial phylogenetic similarity ( $\beta$ diversity) between the groups. Unweighted weighted UniFrac 2-dimensional principal coordinate analysis revealed shifted site-specific clusters from the 3 groups at the phylum level, from the PDS group (red circle) to the PDS + H-BCE group (green circle; Figure 3A). Unfortunately, nonspecific clusters were present in the other groups (PDS + L-BCE and H-BCE; data not 
(A) Unweighted Unifrac

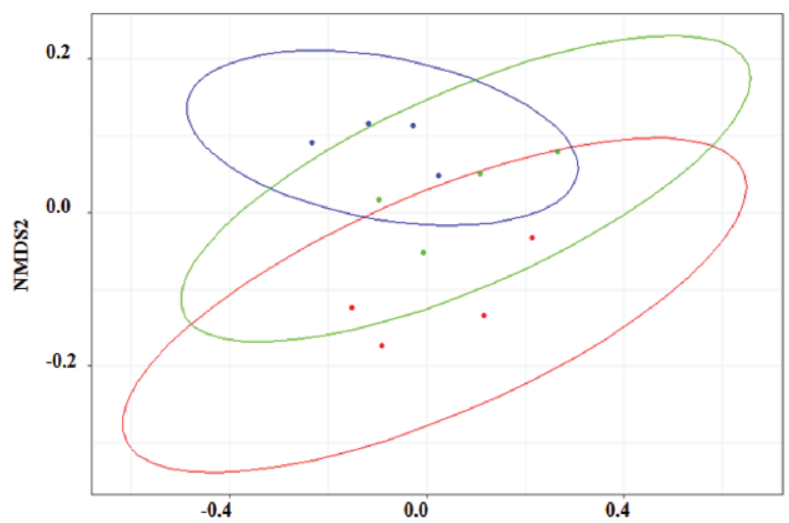

(B)

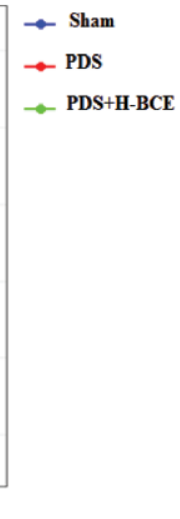

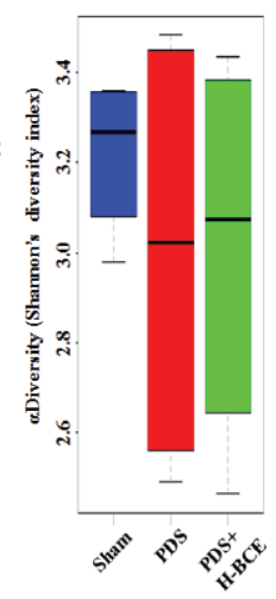

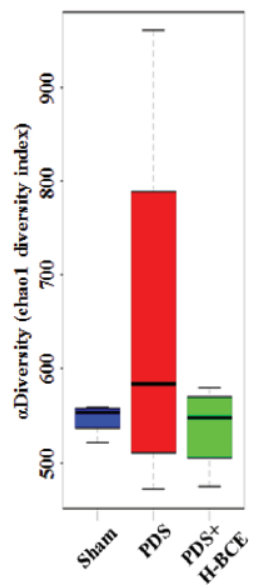

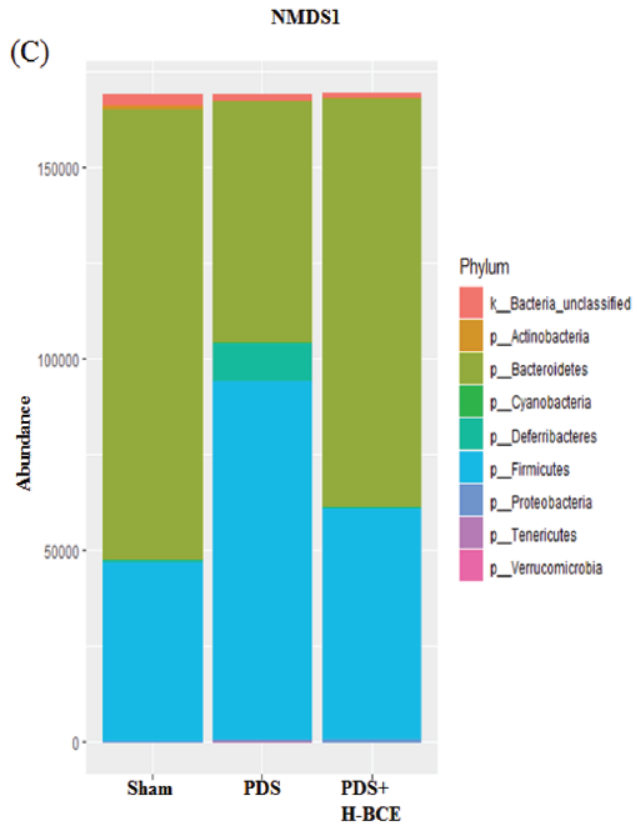

(E)

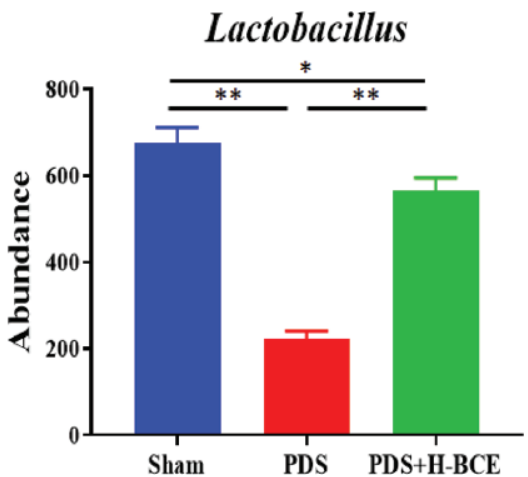

(D)

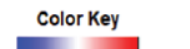

\section{$\log 2$ data}

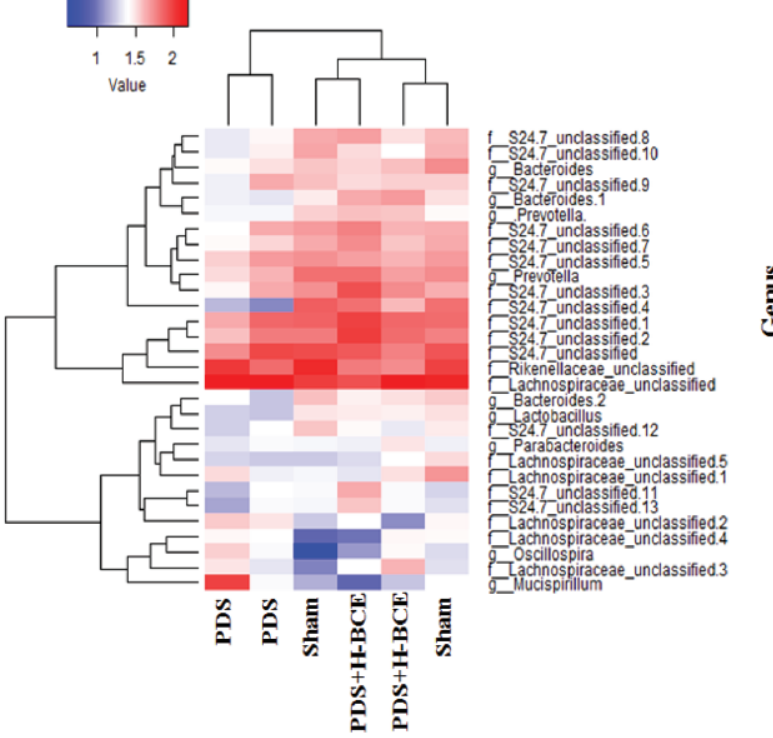

(F)

\section{Bacteroides}

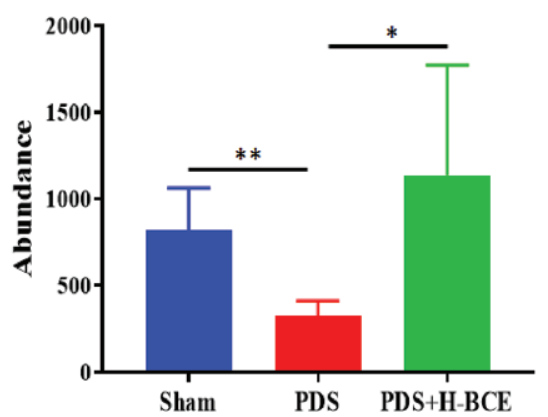

Figure 3. Alterations of gut microbiota caused by bovine colostrum-derived exosome (BCE) ingestion in mice with osteoporosis. (A) Two-dimensional principal coordinate analysis based on unweighted UniFrac distances with site-specific clustering of the 3 groups [sham, osteoporosis-induced (PDS), and PDS + high (H)-BCE, $\mathrm{n}=4$, randomly pooled/group]. Due to nonspecific clustering, the H-BCE and PDS + low (L)-BCE groups are not shown. NMDS = nonmetric multidimensional scaling. (B) Comparison of $\alpha$ diversity indices among the 3 groups. Diversity in the gut bacterial community was measured using the Shannon and Chao-1 indices. Values are expressed as box-and-whisker plots (median, 25th to 75th percentiles, and minimum to maximum values). (C) Community bar-plot analysis showing the community composition and species abundance in the 3 groups. (D) Compositions of microbiota at the genus level by heat map analysis in the 3 groups. (E) and (F) Abundance of Lactobacillus and Bacteroides were compared in 3 representative groups; data are presented as means \pm SEM. Asterisks indicate significant differences between groups $\left(* P<0.05,{ }^{*} P<0.001\right)$. 
shown). The gut microbiota $\alpha$ diversity was analyzed using the Chao1 and Shannon diversity indices. The patterns of these indices tend to be recovered from the PDS group (red bar) to the PDS + H-BCE group (green bar; Figure 3B). Community bar-plot analysis showed the community composition in the 3 groups, suggesting that osteoporosis increased Firmicutes and Deferribacteres, whereas Bacteroidetes were decreased (Figure 3C). The increased Deferribacteres and Bacteroidetes in the PDS group was significantly decreased by BCE. The top 30 abundant genera in the 3 groups (sham, PDS, and PDS + H-BCE) were selected to construct a representative heat map (Figure 3D). Based on the heat map, the relative abundance of Lactobacillus and Bacteroides in the PDS group was decreased more than in the sham group, but that decrease was significantly reversed by $\mathrm{BCE}$ intake (Figure $3 \mathrm{E}$ and $3 \mathrm{~F}$ ).

A previous study reported that Lactobacillus spp. are reduced in the gut microbiota by osteoarthritis (Collins et al., 2015). We observed a similar reduction in Lactobacillus in the PDS group in this study. The gut microbiota may influence bone metabolism, but the exact mechanism remains unclear. Many studies have reported that probiotics can relieve osteoporosis (Collins et al., 2017), and that Lactobacillus reuteri prevents bone loss in mice (McCabe et al., 2013; Britton et al., 2014). In addition, Lactobacillus rhamnosus GG and the commercial mixture VSL\#3, which are well known as probiotics, also prevent bone loss in ovariectomized mice (Li et al., 2016). Further, Bifidobacterium longum alleviated femoral bone loss and increased BMD in ovariectomized rats (Parvaneh et al., 2015), supporting the notion that probiotic bacteria could alleviate bone loss in osteoporosis by improving intestinal microbial constitution.

Recently, Zhou et al. (2019) demonstrated that milk exosomes change microbial communities in mice through exosomal RNA content, suggesting that exosomes and their cargos (mainly RNA contents) participate in the interkingdom communication between bacteria and animals. Teng et al. (2018) showed that the anti-inflammatory function of plant-derived exosomes changes the gut microbial community and regulates bacterial gene expression via exosomal microRNA (Teng et al., 2018). Exosomes are much richer in milk than in other agricultural products, so milk could prevent degenerative diseases. However, the role of exosomes in milk, especially exosomal microRNA, has yet to be determined. Currently, some microRNA are being evaluated as delivery systems for osteoporosis and bone fracture treatments (Sun et al., 2019). The microRNA, miR-30a, miR-92a, miR-26, and miR-21 are important RNA species that are abundant in milk, and likely play important biological roles in bone tissue remodeling.
Further investigation between milk exosome-derived microRNA and target mRNA that encode osteoporosisrelated proteins is needed. Recent reports suggest that milk-derived exosomes alter microbial communities in mice, possibility via cell-to-cell communication (Zhou et al., 2019).

Our study provides new insights into BCE action on glucocorticoid-induced loss of bone mass, micro-architectural integrity, and alterations of gut microbiota by the diet. In-depth studies on BCE components, such as protein and microRNA, are required to understand how $\mathrm{BCE}$ function at the molecular level. Haffner-Luntzer et al. (2016) describes limitations in osteoporotic mouse models, due to significant biological differences between mice and humans. This study also has some limitations related to using a mouse model, but we suggest $\mathrm{BCE}$ as a new alternative for osteoporosis treatment because microRNA are highly homologous and likely act in interkingdom regulation.

\section{ACKNOWLEDGMENTS}

This research was supported by the High Value-Added Food Technology Development Program of the Korean Institute of Planning and Evaluation for Technology in Food, Agriculture, Forestry, and Fisheries (iPET), the Ministry for Food, Agriculture, Forestry, and Fisheries of the Republic of Korea (Gwacheon, Korea; 318090-031-WT011), and the National Research Foundation of Korea Grant funded by the Korean government (Seoul, Korea; NRF-2018R1D1A3B07050304). All authors declare that (i) no support, financial or otherwise, has been received from any organization that may have an interest in the submitted work; and (ii) there are no other relationships or activities that could appear to have influenced the submitted work.

\section{REFERENCES}

Arntz, O. J., B. C. Pieters, M. C. Oliveira, M. G. Broeren, M. B. Bennink, M. de Vries, P. L. van Lent, M. I. Koenders, W. B. van den Berg, P. M. van der Kraan, and F. A. J. van de Loo. 2015. Oral administration of bovine milk derived extracellular vesicles attenuates arthritis in two mouse models. Mol. Nutr. Food Res. 59:1701-1712. https://doi.org/10.1002/mnfr.201500222.

Bang, C., and T. Thum. 2012. Exosomes: New players in cell-cell communication. Int. J. Biochem. Cell Biol. 44:2060-2064. https://doi .org/10.1016/j.biocel.2012.08.007.

Batty, B. S., and M. Bionaz. 2019. Graduate Student Literature Review: The milk behind the mustache: A review of milk and bone biology. J. Dairy Sci. 102:7608-7617. https://doi.org/10.3168/jds .2019-16421.

Britton, R. A., R. Irwin, D. Quach, L. Schaefer, J. Zhang, T. Lee, N. Parameswaran, and L. R. McCabe. 2014. Probiotic L. reuteri treatment prevents bone loss in a menopausal ovariectomized mouse model. J. Cell. Physiol. 229:1822-1830. https://doi.org/10 $.1002 /$ jcp. 24636 .

Collins, F. L., N. D. Rios-Arce, J. D. Schepper, N. Parameswaran, and L. R. McCabe. 2017. The potential of probiotics as a therapy 
for osteoporosis. Microbiol. Spectr. 5:213-233. https://doi.org/10 1128/microbiolspec.BAD-0015-2016.

Collins, K. H., H. Paul, R. Reimer, R. Seerattan, D. Hart, and W. Herzog. 2015. Relationship between inflammation, the gut microbiota, and metabolic osteoarthritis development: Studies in a rat model. Osteoarthritis Cartilage 23:1989-1998. https://doi.org/10 .1016/j.joca.2015.03.014.

Danneskiold-Samsøe, N. B., H. Dias de Freitas Queiroz Barros, R. Santos, J. L. Bicas, C. B. B. Cazarin, L. Madsen, K. Kristiansen, G. M. Pastore, S. Brix, and M. R. Maróstica Júnior. 2019. Interplay between food and gut microbiota in health and disease. Food Res. Int. 115:23-31. https://doi.org/10.1016/j.foodres.2018 .07 .043 .

DeSantis, T. Z., P. Hugenholtz, N. Larsen, M. Rojas, E. L. Brodie, K. Keller, T. Huber, D. Dalevi, P. Hu, and G. L. Andersen. 2006. Greengenes, a chimera-checked 16S rRNA gene database and workbench compatible with ARB. Appl. Environ. Microbiol. 72:5069-5072. https://doi.org/10.1128/AEM.03006-05.

Ercolini, D., and V. Fogliano. 2018. Food design to feed the human gut microbiota. J. Agric. Food Chem. 66:3754-3758. https://doi.org/ 10.1021/acs.jafc.8b00456.

Frost, H. M., J. Ferretti, and W. Jee. 1998. Perspectives: Some roles of mechanical usage, muscle strength, and the mechanostat in skeletal physiology, disease, and research. Calcif. Tissue Int. 62:1-7. https://doi.org/10.1007/s002239900384.

Ghayor, C., R. M. Correro, K. Lange, L. S. Karfeld-Sulzer, K. W. Grätz, and F. E. Weber. 2011. Inhibition of osteoclast differentiation and bone resorption by N-methylpyrrolidone. J. Biol. Chem. 286:24458-24466. https://doi.org/10.1074/jbc.M111.223297.

Guss, J. D., M. W. Horsfield, F. F. Fontenele, T. N. Sandoval, M. Luna, F. Apoorva, S. F. Lima, R. C. Bicalho, A. Singh, R. E. Ley, M. C. H. van der Meulen, S. R. Goldring, and C. J. Hernandez. 2017. Alterations to the gut microbiome impair bone strength and tissue material properties. J. Bone Miner. Res. 32:1343-1353. https://doi.org/10.1002/jbmr.3114.

Haffner-Luntzer, M., A. Kovtun, A. E. Rapp, and A. Ignatius. 2016. Mouse models in bone fracture healing research. Curr. Mol. Biol. Rep. 2:101-111. https://doi.org/10.1007/s40610-016-0037-3.

Hayman, A. R. 2008. Tartrate-resistant acid phosphatase (TRAP) and the osteoclast/immune cell dichotomy. Autoimmunity 41:218-223. https://doi.org/10.1080/08916930701694667.

Ibáñez, L., M. Rouleau, A. Wakkach, and C. Blin-Wakkach. 2019. Gut microbiome and bone. Joint Bone Spine 86:43-47. https://doi.org/ 10.1016/j.jbspin.2018.02.008.

Kumar, D., D. Gupta, S. Shankar, and R. K. Srivastava. 2015. Biomolecular characterization of exosomes released from cancer stem cells: Possible implications for biomarker and treatment of cancer. Oncotarget 6:3280. https://doi.org/10.18632/oncotarget.2462.

Leder, B. Z., J. N. Tsai, A. V. Uihlein, P. M. Wallace, H. Lee, R. M. Neer, and S.-A. M. J. T. L. Burnett-Bowie. 2015. Denosumab and teriparatide transitions in postmenopausal osteoporosis (the DATA-Switch study): Extension of a randomised controlled trial. Lancet 386:1147-1155. https://doi.org/10.1016/S0140-6736(15)61120 -5 .

Li, J.-Y., B. Chassaing, A. M. Tyagi, C. Vaccaro, T. Luo, J. Adams, T. M. Darby, M. N. Weitzmann, J. G. Mulle, A. T. Gewirtz, R. M. Jones, and R. Pacifici. 2016. Sex steroid deficiency-associated bone loss is microbiota dependent and prevented by probiotics. J. Clin. Invest. 126:2049-2063. https://doi.org/10.1172/JCI86062.

Maburutse, B. E., M.-R. Park, S. Oh, and Y. Kim. 2017. Evaluation and characterization of milk-derived microvescicle isolated from bovine colostrum. Korean J. Food Sci. Anim. Resour. 37:654-662. https://doi.org/10.5851/kosfa.2017.37.5.654.

Marchesi, J. R., D. H. Adams, F. Fava, G. D. Hermes, G. M. Hirschfield, G. Hold, M. N. Quraishi, J. Kinross, H. Smidt, K. M. Tuohy, L. V. Thomas, E. G. Zoetendal, and A. Hart. 2016. The gut microbiota and host health: A new clinical frontier. Gut 65:330-339. https:// doi.org/10.1136/gutjnl-2015-309990.

McCabe, L. R., R. Irwin, L. Schaefer, and R. A. Britton. 2013. Probiotic use decreases intestinal inflammation and increases bone density in healthy male but not female mice. J. Cell. Physiol. 228:1793-1798. https://doi.org/10.1002/jcp.24340.

Michaëlsson, K., A. Wolk, S. Langenskiöld, S. Basu, E. W. Lemming, H. Melhus, and L. Byberg. 2014. Milk intake and risk of mortality and fractures in women and men: Cohort studies. BMJ 349(oct27 1):g6015. https://doi.org/10.1136/bmj.g6015.

Newa, M., K. H. Bhandari, L. Tang, R. Kalvapalle, M. Suresh, and M. R. Doschak. 2011. Antibody-mediated "universal" osteoclast targeting platform using calcitonin as a model drug. Pharm. Res. 28:1131-1143. https://doi.org/10.1007/s11095-011-0376-y.

NIH Consensus Development Panel on Osteoporosis Prevention, Diagnosis, and Therapy. 2001. Osteoporosis prevention, diagnosis, and therapy. JAMA 285:785-795. https://doi.org/10.1001/jama .285.6.785.

Oliveira, M. C., I. Di Ceglie, O. J. Arntz, W. B. Van den Berg, F. H. Van den Hoogen, A. V. Ferreira, P. L. Van Lent, and F. A. Van De Loo. 2017. Milk-derived nanoparticle fraction promotes the formation of small osteoclasts but reduces bone resorption. J. Cell. Physiol. 232:225-233. https://doi.org/10.1002/jcp.25414.

Parvaneh, K., M. Ebrahimi, M. R. Sabran, G. Karimi, A. N. M. Hwei, S. Abdul-Majeed, Z. Ahmad, Z. Ibrahim, and R. Jamaluddin. 2015. Probiotics (Bifidobacterium longum) increase bone mass density and upregulate Sparc and Bmp-2 genes in rats with bone loss resulting from ovariectomy. BioMed Res. Int. 2015:897639.

Pruesse, E., C. Quast, K. Knittel, B. M. Fuchs, W. Ludwig, J. Peplies, and F. O. Glöckner. 2007. SILVA: A comprehensive online resource for quality checked and aligned ribosomal RNA sequence data compatible with ARB. Nucleic Acids Res. 35:7188-7196. https:// doi.org/10.1093/nar/gkm864.

Rani, P., V. R. Yenuganti, S. Shandilya, S. K. Onteru, and D. Singh. 2017. miRNAs: The hidden bioactive component of milk. Trends Food Sci. Technol. 65:94-102. https://doi.org/10.1016/j.tifs.2017 .05 .007 .

Rognes, T., T. Flouri, B. Nichols, C. Quince, and F. Mahé. 2016 VSEARCH: A versatile open source tool for metagenomics. PeerJ 4:e2584. https://doi.org/10.7717/peerj.2584.

Samuel, M., D. Chisanga, M. Liem, S. Keerthikumar, S. Anand, C.S. Ang, C. G. Adda, E. Versteegen, M. Jois, and S. Mathivanan. 2017. Bovine milk-derived exosomes from colostrum are enriched with proteins implicated in immune response and growth. Sci. Rep. 7:5933. https://doi.org/10.1038/s41598-017-06288-8.

Schloss, P. D., S. L. Westcott, T. Ryabin, J. R. Hall, M. Hartmann, E. B. Hollister, R. A. Lesniewski, B. B. Oakley, D. H. Parks, C. J. Robinson, J. W. Sahl, B. Stres, G. G. Thallinger, D. J. Van Horn, and C. F. Weber. 2009. Introducing mothur: Open-source, platform-independent, community-supported software for describing and comparing microbial communities. Appl. Environ. Microbiol. 75:7537-7541. https://doi.org/10.1128/AEM.01541-09.

Sjögren, K., C. Engdahl, P. Henning, U. H. Lerner, V. Tremaroli, M. K. Lagerquist, F. Bäckhed, and C. Ohlsson. 2012. The gut microbiota regulates bone mass in mice. J. Bone Miner. Res. 27:13571367. https://doi.org/10.1002/jbmr.1588.

Soysa, N. S., and N. Alles. 2016. Osteoclast function and bone-resorbing activity: An overview. Biochem. Biophys. Res. Commun. 476:115-120. https://doi.org/10.1016/j.bbrc.2016.05.019.

Sun, Q., X. Chen, J. Yu, K. Zen, C.-Y. Zhang, and L. Li. 2013. Immune modulatory function of abundant immune-related microR NAs in microvesicles from bovine colostrum. Protein Cell 4:197210. https://doi.org/10.1007/s13238-013-2119-9.

Sun, X., Q. Guo, W. Wei, S. Robertson, Y. Yuan, and X. Luo. 2019. Current progress on microRNA-based gene delivery in the treatment of osteoporosis and osteoporotic fracture. Int. J. Endocrinol. 2019:6782653. https://doi.org/10.1155/2019/6782653.

Teng, Y., Y. Ren, M. Sayed, X. Hu, C. Lei, A. Kumar, E. Hutchins, J. $\mathrm{Mu}$, Z. Deng, C. Luo, K. Sundaram, M. K. Sriwastva, L. Zhang, M. Hsieh, R. Reiman, B. Haribabu, J. Yan, V. R. Jala, D. M. Miller, K. Van Keuren-Jensen, M. L. Merchant, C. J. McClain, J. W. Park, N. K. Egilmez, and H.-G. Zhang. 2018. Plant-derived exosomal microRNAs shape the gut microbiota. Cell Host Microbe 24:637-652. https://doi.org/10.1016/j.chom.2018.10.001. 
Thiele, S., U. Baschant, A. Rauch, and M. Rauner. 2014. Instructions for producing a mouse model of glucocorticoid-induced osteoporosis. Bonekey Rep. 3:552. https://doi.org/10.1038/bonekey.2014.47.

Uenishi, K. 2006. Prevention of osteoporosis by foods and dietary supplements. Prevention of osteoporosis by milk and dairy products. Clin. Calcium 16:1606-1614.

Uenishi, K., H. Ishida, Y. Toba, S. Aoe, A. Itabashi, and Y. Takada. 2007. Milk basic protein increases bone mineral density and improves bone metabolism in healthy young women. Osteoporos. Int 18:385-390. https://doi.org/10.1007/s00198-006-0228-5.

Unno, T. 2015. Bioinformatic suggestions on MiSeq-based microbial community analysis. J. Microbiol. Biotechnol. 25:765-770. https:/ /doi.org/10.4014/jmb.1409.09057.

Uruakpa, F., M. Ismond, and E. Akobundu. 2002. Colostrum and its benefits: A review. Nutr. Res. 22:755-767. https://doi.org/10 .1016/S0271-5317(02)00373-1.

Weaver, C. M., D. Alexander, C. Boushey, B. Dawson-Hughes, J. Lappe, M. LeBoff, S. Liu, A. Looker, T. Wallace, and D. Wang. 2016. Calcium plus vitamin D supplementation and risk of fractures: An updated meta-analysis from the National Osteoporosis Foundation. Osteoporos. Int. 27:367-376. https://doi.org/10 .1007/s00198-015-3386-5.

Zempleni, J., S. Sukreet, F. Zhou, D. Wu, and E. Mutai. 2019. Milkderived exosomes and metabolic regulation. Annu. Rev. Anim. Biosci. 7:245-262. https://doi.org/10.1146/annurev-animal $-020518-115300$.
Zheng, H., X. Yu, P. Collin-Osdoby, and P. Osdoby. 2006. RANKL stimulates inducible nitric-oxide synthase expression and nitric oxide production in developing osteoclasts. An autocrine negative feedback mechanism triggered by RANKL-induced interferon- $\beta$ via NF- $\kappa \mathrm{B}$ that restrains osteoclastogenesis and bone resorption. J. Biol. Chem. 281:15809-15820. https://doi.org/10.1074/jbc .M513225200.

Zhou, F., H. A. Paz, M. Sadri, J. Cui, S. D. Kachman, S. C. Fernando, and J. Zempleni. 2019. Dietary bovine milk exosomes elicit changes in bacterial communities in C57BL/6 mice. Am. J. Physiol. Gastrointest. Liver Physiol. 317:G618-G624. https://doi.org/10 .1152/ajpgi.00160.2019.

Zhou, Q., M. Li, X. Wang, Q. Li, T. Wang, Q. Zhu, X. Zhou, X. Wang, X. Gao, and X. Li. 2012. Immune-related microRNAs are abundant in breast milk exosomes. Int. J. Biol. Sci. 8:118-123. https:// doi.org/10.7150/ijbs.8.118.

\section{ORCIDS}

M. Kang ๑ https://orcid.org/0000-0002-2366-7970

M. R. Park (1) https://orcid.org/0000-0002-0149-743X

Y. Kim ৫ https://orcid.org/0000-0001-6769-0657

S. Oh (1) https://orcid.org/0000-0002-2428-412X 